\title{
A 0.1-to-1.2GHz Tunable 6th-Order N-Path Channel-Select Filter with 0.6dB Passband Ripple and $+7 \mathrm{dBm}$ Blocker Tolerance
}

\section{Milad Darvishi, Ronan van der Zee, Bram Nauta \\ University of Twente, Enschede, The Netherlands}

Radio receivers should be robust to large out-of-band blockers with small degradation in their sensitivity. N-path mixers can be used as mixer-first-receivers [1] with good linearity and RF filtering [2]. However, 1/f noise calls for large active device sizes for IF circuits and high power consumption. The 1/f noise issue can be relaxed by having RF gain. However, to avoid desensitization by large out-of-band blockers, a bandpass filter (BPF) with sharp cut-off frequency is required in front of the RF amplifiers. $g_{m}-C$ BPFs suffer from tight tradeoffs among DR, power consumption, $Q$ and $f_{c}$. Also, on-chip $Q-$ enhanced LC BPFs [3] are not suitable due to low DR, high area consumption and non-tunability. Therefore, bulky and non-tunable SAW filters are used. N-path BPFs offer high $Q$ while their center frequency is tuned by the clock frequency [2]. Compared to $\mathrm{g}_{\mathrm{m}}-\mathrm{C}$ filters, this technique decouples the required $Q$ from the $D R$. The 4-path filter in [4] has only $2^{\text {nd }}$ order filtering and limited rejection. The order and rejection of N-path BPFs can be increased by cascading [5], but this renders a "round" passband shape. The $4^{\text {th }}$ order 4-path BPF in [6] has a "flat" pass-band shape and high rejection but a high NF. This work solves the noise issue of [6] while achieving the same out-of-band linearity and adding $25 \mathrm{~dB}$ of voltage gain to relax the noise requirement of the subsequent stages.

We propose a low-noise tunable $6^{\text {th }}$ order N-path BPF with Chebyshev shape, high $Q$, high out-of-band linearity and high stop-band rejection. The idea is to substitute each LC tank in a capacitor-coupledresonator filter with its $\mathrm{N}$-path counterpart. However, this idea is not applicable due to the frequency dependency of the coupling networks. Instead, gyrators are used as coupling networks due to their wideband nature. A conventional singly-terminated $6^{\text {th }}$ order LC BPF prototype is shown in Fig.1.a. The series LC tank can be converted into a parallel one by using two gyrators (Fig.1.b). The first gyrator $\mathrm{G}_{1}$ is substituted by a single $g_{m}$ cell to simplify the design by stagger tuning a $2^{\text {nd }}$ and a $4^{\text {th }}$ order BPF (Fig.1.b). Each LC tank can be emulated by an 8-path switched-capacitor filter shown in Fig.1.c [2]. In $\mathrm{N}$-path filters, signals at $\left|(k N-1) f_{10}\right| k=1,2, .$. fold back to the pass-band of the filter [2]. Utilization of 8 phases greatly relaxes the folding-back issue. Each 8-path switched-capacitor bank is differential to suppress BPF transfer functions at even harmonics of the $f_{10}$. Therefore, the next BPF transfer function will be at $3 f_{10}$. To save area, also each tank capacitor is differential. The schematic of the proposed filter is shown in Fig.2. The gyrator $\mathrm{G}_{2}$ is similar to [7], but by making the feedforward path of the gyrator stronger than its feedback path while keeping the gyration ratio constant, the noise contribution of the 
gyrator is reduced while the gain of the filter is increased. Each $g_{m}$ cell is a self-biased inverter. Switch resistances can de-Q the filter and therefore they should be taken into account in the design process. The extra phase shifts due to parasitic capacitors at node $x$ and $V_{\text {out }}$ of the filter distort the pass-band shape of the filter and cause more than $1.5 \mathrm{~dB}$ of peaking. A miller-capacitor $\mathrm{C}_{f}$ is exploited to slightly lower the $f_{c}$ of the first resonator while enhancing the bandwidth at $V_{\text {out }}$ to reduce the effect of parasitic capacitors on the filter. The simulated effect is shown in Fig.3.a. The filter can achieve excellent out-ofband linearity because of: 1 ) the first section being "passive" (so the first $g_{m}$ already receives a $2^{\text {nd }}$ order filtered signal) and the further filtering in the subsequent stages; 2) the very linear differential I/V characteristic of an inverter when loaded with low impedance. The filter can attain a low NF because of: 1) the exploitation of asymmetric gyrators; 2) a relatively high value of $g_{\mathrm{m} 1}$; 3 ) the utilization of a very small amount of negative admittance at node $x$ and $V_{\text {out; }}$ 4) a low switch resistance; and 5) the usage of 8 phases (less harmonic folding of noise).

The filter was realized in $65 \mathrm{~nm}$ LP CMOS. The values of $\mathrm{g}_{\mathrm{m} 1}, \mathrm{~g}_{\mathrm{m} 2}$ and $\mathrm{g}_{\mathrm{m} 3}$ are $60 \mathrm{mS}, 24 \mathrm{mS}$ and $4 \mathrm{mS}$ respectively and made with minimum channel length transistors. $C_{1}, C_{2}$ and $C_{3}$ are $63 p F, 53 p F$ and $43 \mathrm{pF}$, respectively. Each tank capacitor is a combination of MOM and accumulation-mode NMOS capacitors to increase its density. The $50 \Omega$ measurement interface is depicted in Fig.3.b. Two commondrain buffers are used for $S_{21}, N F, \| P_{3}(\mathrm{OOB})$ and $\mathrm{B}_{1 \mathrm{~dB}, \mathrm{CP}}$ measurements at port I. To measure the inband linearity while not being affected by the buffers, a resistive voltage divider is used at port II. The switches are made of NMOS transistors with an on-resistance of $10 \Omega$. The source and drain of each switch is biased to roughly $0.6 \mathrm{~V}$ due to the self-biased inverters. The clock signals are ac-coupled to the gate of each switch which has a high ohmic resistor to a bias voltage of $0.75 \mathrm{~V}$. A divide-by-8 frequency divider is used to achieve 8 non-overlapping clocks. It should be noted that if the capacitor voltages of the third stage are taken as baseband outputs, the filter can be used as a receiver, taking benefit of the filtering properties. Because of its simplicity, the filter is easily portable to new CMOS generations.

The chip photo is shown in Fig.7 with an active area of $0.27 \mathrm{~mm}^{2}$. The tuneability of the filter from $0.1 \mathrm{GHz}-1.2 \mathrm{GHz}$ is shown in Fig.4. The pass-band ripple of the filter is $<0.6 \mathrm{~dB}$. The negative resistances $R_{\text {neg1,2 }}$ are slightly changed by tuning their supply voltages ( $<8 \%$ ) over the tuning range, however without any modifications, the ripple is still less than $1 \mathrm{~dB}(<0.5 \mathrm{~dB}$ in the simulation) over the tuning range. Due to the use of small amount of negative admittance, the filter sensitivity to negative resistance values compared to conventional $\mathrm{g}_{\mathrm{m}} \mathrm{C}$ filters [7] is reduced. The stop-band rejection of the filter is $59 \mathrm{~dB}$. The gain of the filter is $25 \mathrm{~dB}$ (de-embedding the loss of buffers) and its bandwidth is $8 \mathrm{MHz}$. The $S_{11}$ varies between $-5 \mathrm{~dB}$ and $-8 \mathrm{~dB}$, in the whole band. The measured NF for different $f_{10}$ is shown in Fig.5.a and is $2.8 \mathrm{~dB}$ on average. The measured $\| P_{3}(\mathrm{OOB})$ and $\mathrm{B}_{1 \mathrm{~dB}, \mathrm{CP}}$ for different offset 
frequencies from $f_{l o}$ is shown in Fig.5.b. The measured $\|_{3}(\mathrm{OOB})$ of $+26 \mathrm{dBm}$ and $1 \mathrm{~dB}$ blocker compression point $\mathrm{B}_{1 \mathrm{~dB}, \mathrm{CP} \text { of }}+7 \mathrm{dBm}$ are achieved at $\Delta \mathrm{f}=50 \mathrm{MHz}$. The measured transfer function of the filter at $f_{10}=1 \mathrm{GHz}$ with and without a blocker with $P_{\text {in }}=2.3 \mathrm{dBm}$ located at $\Delta f=20 \mathrm{MHz}$ from the center frequency, is shown in Fig.5.c. The filter draws $11.7 \mathrm{~mA}$ and the LO chain draws 3 to $36 \mathrm{~mA}$ from $1.2 \mathrm{~V}$ in the tuning range. The LO feedtrough to the input port is less than $-64 \mathrm{dBm}$. The folding-back starts only from $7 f l o$. However due to the mismatch, folding occurs at $3 f_{10}$ and $5 f_{10}$ with measured normalized gain of $-54 \mathrm{~dB}$ and $-68 \mathrm{~dB}$, respectively.

The filter is compared with state of the art integrated filters [3-4,6] and complete receivers [5,8-9] in Fig.6. Compared to [4], much better pass-band shape, selectivity and stop-band rejection are obtained. Compared to [5], better out-of-band linearity, filter shape and NF are accomplished. Compared to [6], the NF is improved by more than $7 \mathrm{~dB}$. To our knowledge, this is the first integrated filter that can be used as a channel-select SAW-LNA hybrid which is tunable over a decade in frequency.

\section{Acknowledgements:}

This research is supported by STW. We thank STMicroelectronics for Silicon donation and CMP for assistance. Also thanks go to G. Wienk, H. de Vries and M. Oude Alink.

References:

[1] M. Soer, et al., "A 0.2-to-2.0GHz 65nm CMOS receiver without LNA achieving $>11 \mathrm{dBm} \| \mathrm{IP}_{3}$ and $<6.5$ dB NF", ISSCC Dig. Tech. Papers, pp. 222-223, Feb. 2009

[2] L. Franks and I. Sandberg, "An alternative approach to the realizations of network functions: N-path filter", Bell Syst. Tech. J., pp. 1321-1350, 1960.

[3] T. Soorapanth, S.S. Wong, "A 0-dB IL 2140 $\pm 30 \mathrm{MHz}$ bandpass filter utilizing Q-enhanced spiral inductors in standard CMOS", J. Solid-State Circuits, pp. 579-586, May 2002

[4] A. Ghaffari, E. Klumperink, M.C.M. Soer, B. Nauta, "Tunable High-Q N-Path Band-Pass Filters: Modeling and Verification", J. Solid-State Circuits, pp. 998-1010, May 2011

[5] A. Mirzaei, H. Darabi, D. Murphy, "A Low-Power Process-Scalable Super-Heterodyne Receiver With Integrated High-Q Filters", J. Solid-State Circuits, pp. 2920-2932, Dec. 2011

[6] M. Darvishi, R. Van der Zee, E. Klumperink, B. Nauta, "A 0.3-to-1.2GHz tunable $4^{\text {th }}$-order switched $\mathrm{g}_{\mathrm{m}} \mathrm{C}$ bandpass filter with $>55 \mathrm{~dB}$ ultimate rejection and out-of-band IIP 3 of $+29 \mathrm{dBm}$ ", ISSCC Dig. Tech. Papers, pp. 358-360, Feb. 2012 
[7] B. Nauta, "A CMOS transconductance-C filter technique for very high frequencies", J. Solid-State Circuits, pp. 142-153, Feb. 1992

[8] J. Borremans, et al., "A 40nm CMOS highly linear 0.4-to-6GHz receiver resilient to 0dBm out-ofband blockers", ISSCC Dig. Tech. Papers, pp. 62-64, Feb. 2011

[9] D. Murphy, et al., "A blocker-tolerant wideband noise-cancelling receiver with a 2dB noise figure", ISSCC Dig. Tech. Papers, pp. 74-76, Feb. 2012

\section{Captions:}

Fig.1: a) A conventional singly terminated $6^{\text {th }}$ order LC BPF b) Exploitation of gyrators to transform a series LC tank to parallel one $\mathrm{c}$ ) each LC tank is emulated by a differential switched capacitor 8-path filter

Fig.2: Schematic of the proposed widely tunable $6^{\text {th }}$ order $g_{m}$-switched-C BPF

Fig.3: a) The simulated effect of $C_{f}$ on the transfer function of the filter b) Measurement interface

Fig.4: The transfer function of the filter is tuned with a clock frequency from $100 \mathrm{MHz}$ to $1.2 \mathrm{GHZ}$. The upper figure shows the pass-band details in a span of $20 \mathrm{MHz}$.

Fig.5: a) NF of the filter at different clock frequency; b) $\mathrm{IP}_{3}(\mathrm{OOB})$ and $\mathrm{B}_{1 \mathrm{~dB}, \mathrm{CP}}$ for different offset frequencies; c) Transfer function of the filter at $f_{10}=1 \mathrm{GHz}$ with and without $\mathrm{CW}$ blocker with $P_{b}=2.3 \mathrm{dBm}$ located at $\Delta \mathrm{f}=20 \mathrm{MHz}$ from center frequency

Fig.6: Comparison table

Fig.7: Chip micrograph 


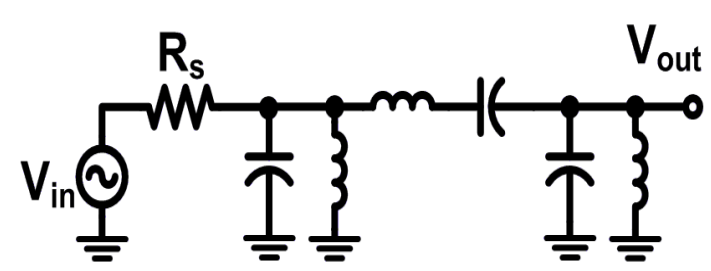

(a)

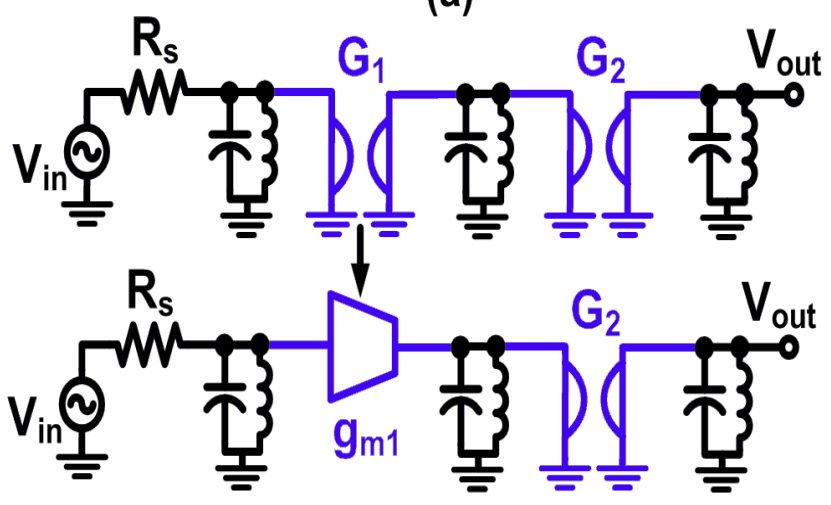

(b)
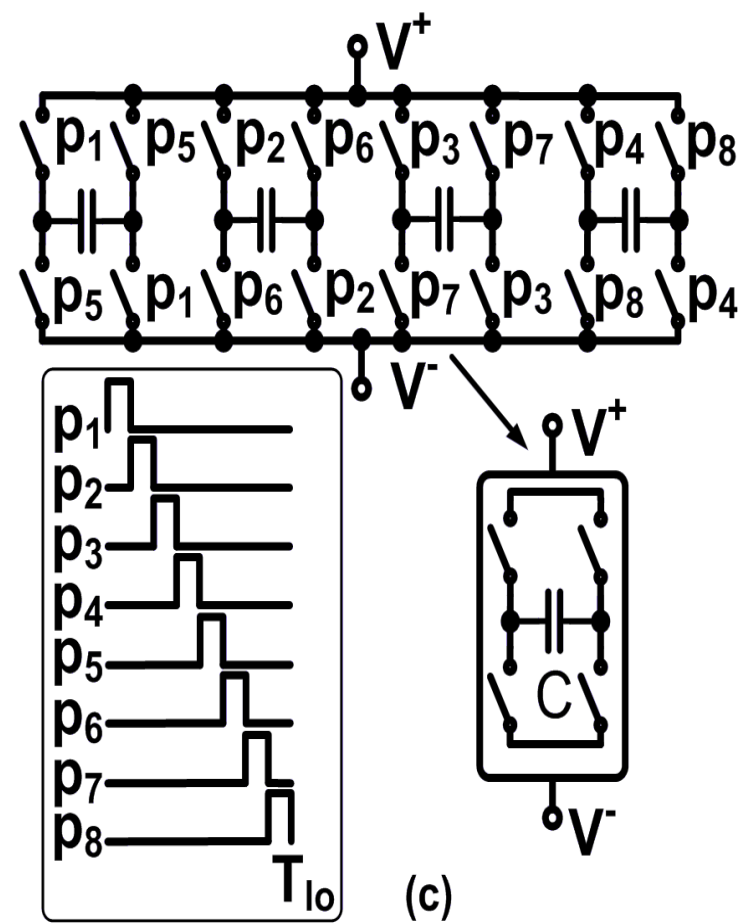

$T_{10}$

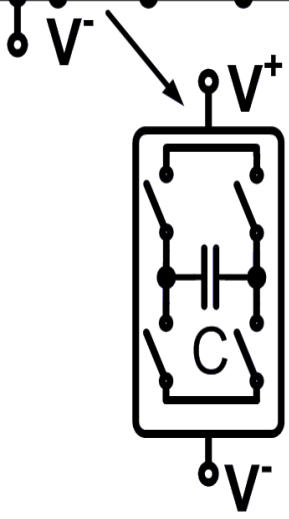

(c)

Fig.1: a) A conventional singly terminated $6^{\text {th }}$ order LC BPF b) Exploitation of gyrators to transform a series LC tank to parallel one c) each LC tank is emulated by a differential switched-capacitor 8-path filter 


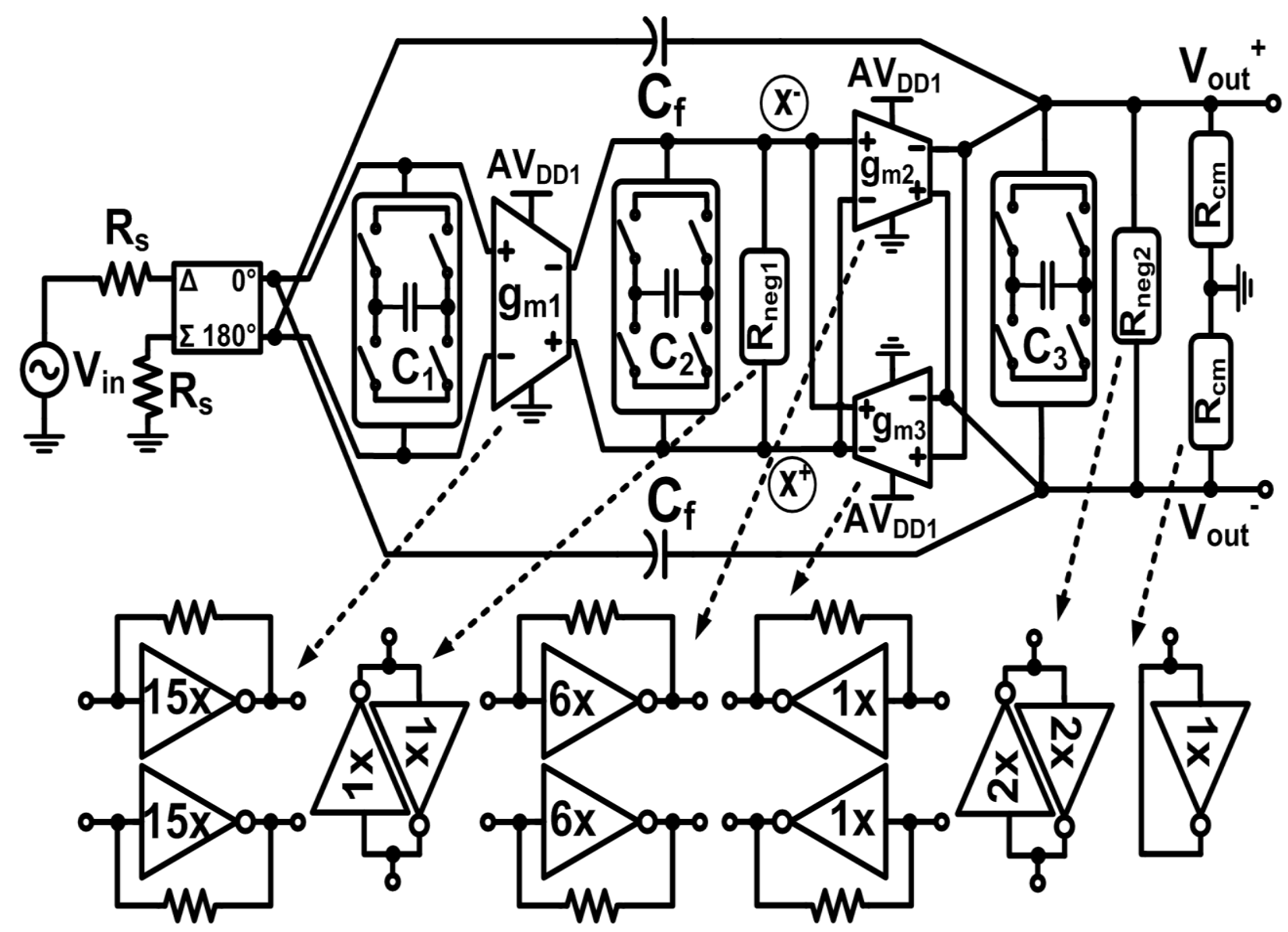

Fig.2: Schematic of a proposed widely tunable $6^{\text {th }}$ order $g_{m}$-switched-C BPF 


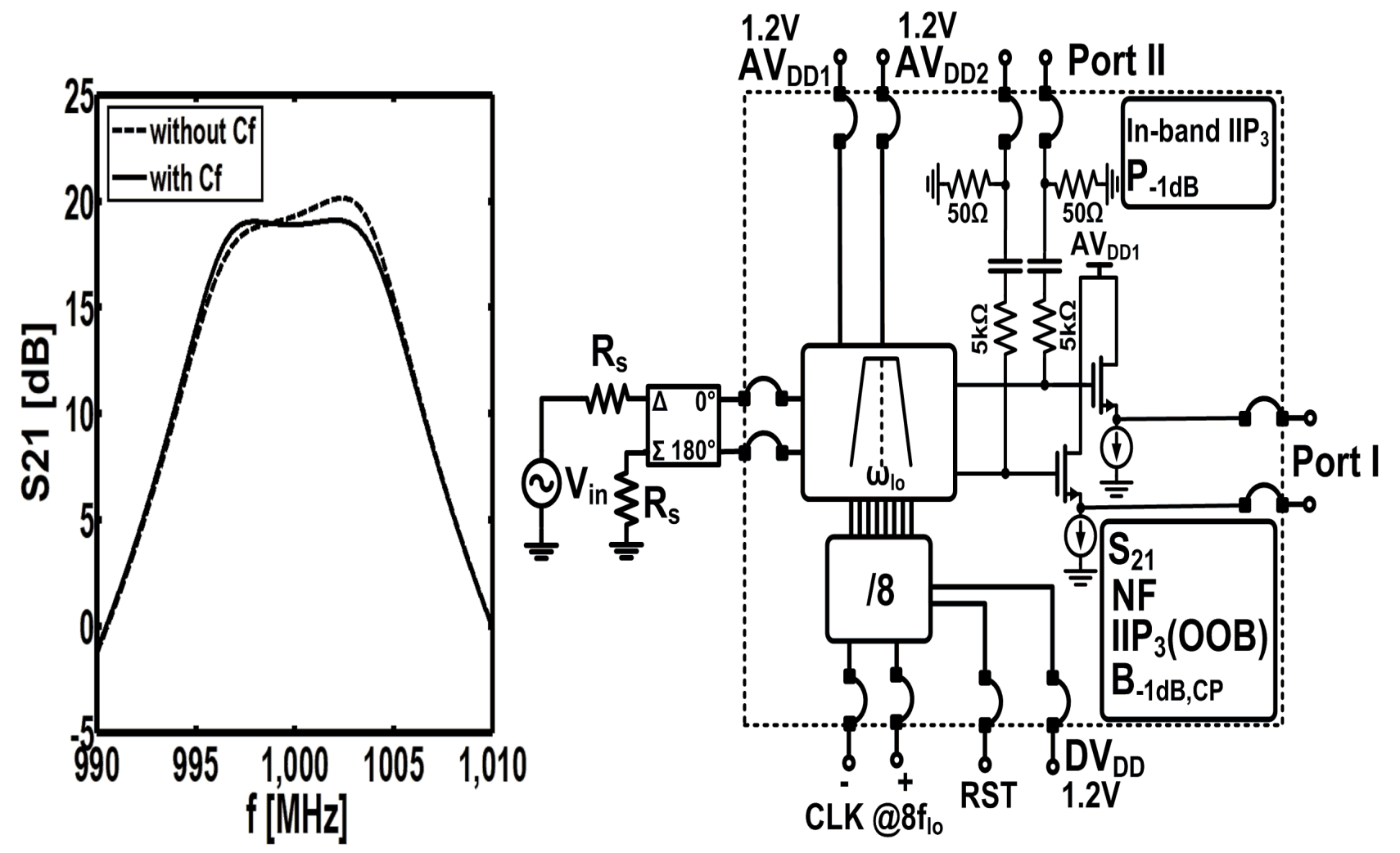

(a)

(b)

Fig.3: a) The simulated effect of $C_{f}$ on the transfer function of the filter b) Measurement interface 


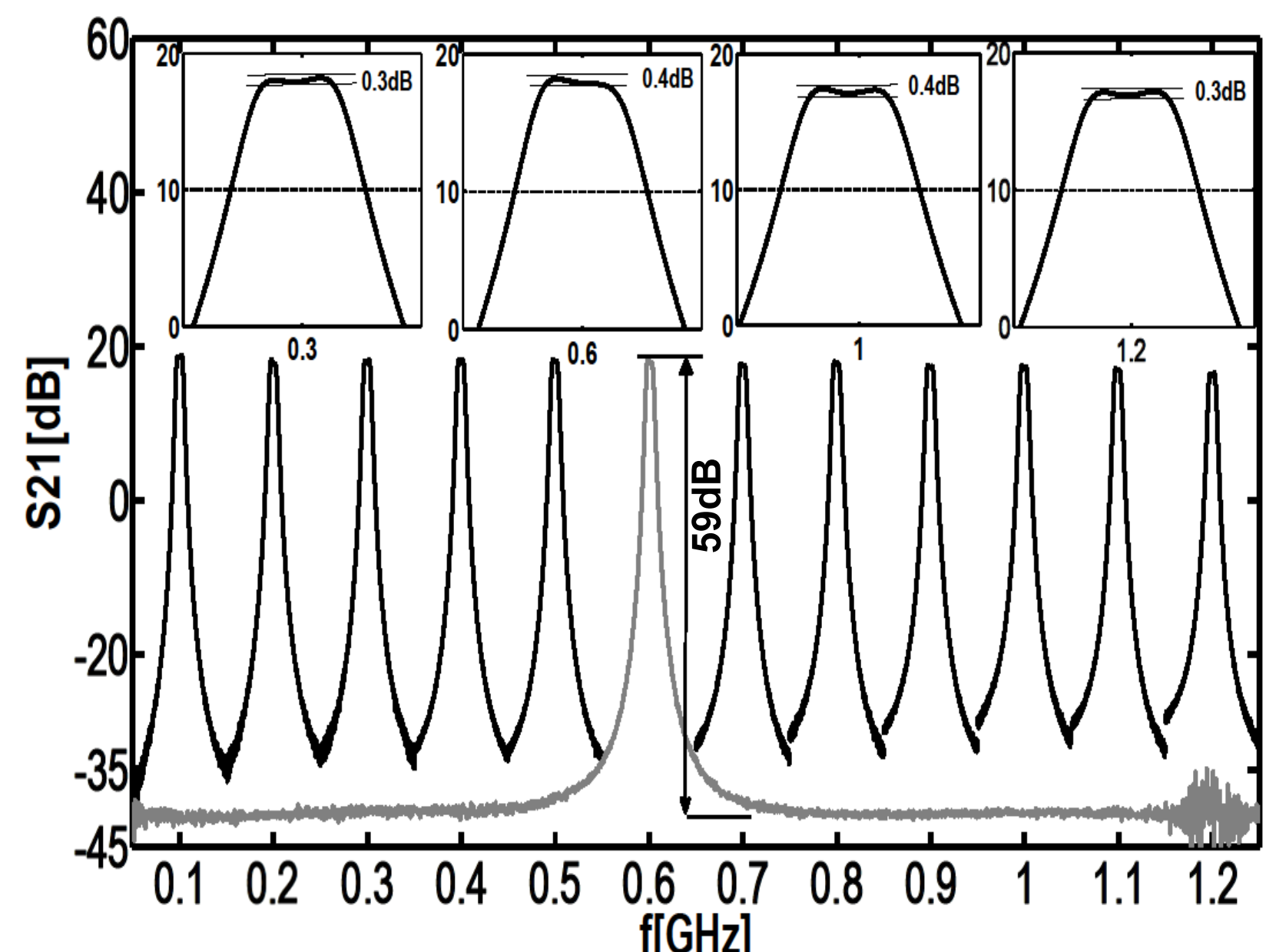

Figure 4: The transfer function of the filter is tuned with a clock frequency from $100 \mathrm{MHz}$ to $1.2 \mathrm{GHz}$. The upper figures show the passband details in a span of $20 \mathrm{MHz}$. 


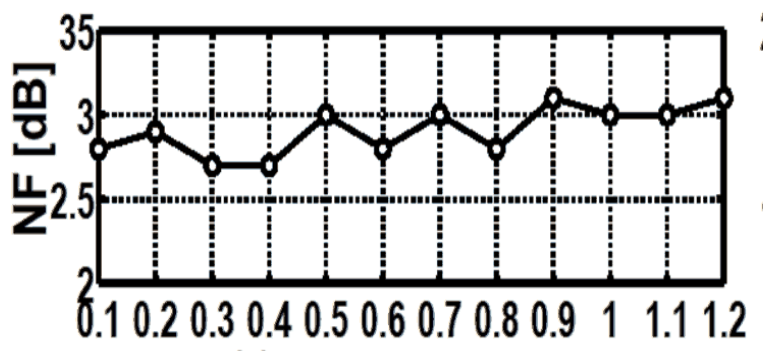

(a) $\mathrm{f}[\mathrm{GHz}]$

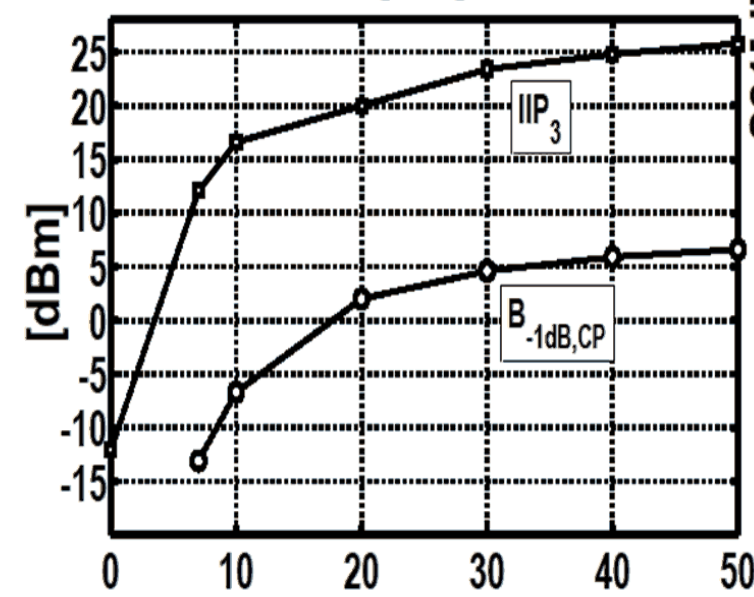

(b) $\Delta f[M H z]$

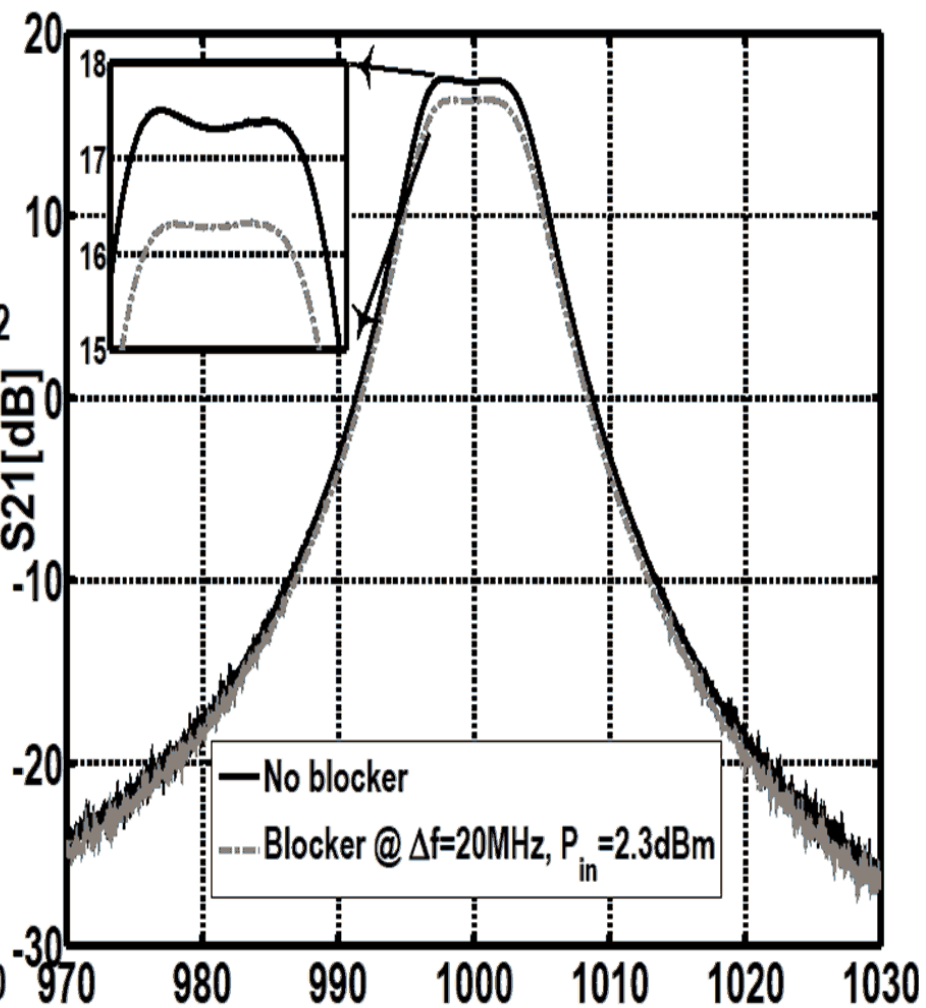

(c) $\mathrm{f}[\mathrm{MHz}]$

Fig.5: a) NF of the filter at different clock frequency; b) $\mathrm{NP}_{3}$ and $B_{1 d B, C P}$ for different offset frequencies; $c$ ) Transfer function of the filter at $f_{10}=1 \mathrm{GHz}$ with and without $\mathrm{CW}$ blocker with $P_{\text {in }}=2.3 \mathrm{dBm}$ located at $\Delta \mathrm{f}=20 \mathrm{MHz}$ from center frequency 


\begin{tabular}{|c|c|c|c|c|c|c|}
\hline & [This work] & $\begin{array}{c}\text { Darvishi } \\
{[6]} \\
\end{array}$ & $\begin{array}{c}\text { Soorapanth } \\
{[3]}\end{array}$ & $\begin{array}{c}\text { Borremans } \\
{[8]}\end{array}$ & $\begin{array}{c}\text { Mirzaei } \\
\text { [5] }\end{array}$ & $\begin{array}{c}\text { Murphy } \\
\text { [9] }\end{array}$ \\
\hline Circuit Type & \multicolumn{3}{|c|}{ Filter } & \multicolumn{3}{|c|}{ Receiver } \\
\hline CMOS Tech. [nm] & 65 & 65 & 250 & 40 & 65 & 40 \\
\hline Frequency range [GHz] & $0.1-1.2$ & $0.4-1.2$ & 2.14 & $0.4-6$ & 2 & $0.08-2.7$ \\
\hline$\| P_{3}(\mathrm{OOB})[\mathrm{dBm}]$ & +26 & +28 & $\mathrm{~N} / \mathrm{A}$ & +10 & -6.3 & +13.5 \\
\hline $\mathrm{B}_{1 \mathrm{~dB}, \mathrm{CP}}[\mathrm{dBm}]$ & +7 & $\mathrm{~N} / \mathrm{A}$ & $\mathrm{N} / \mathrm{A}$ & -8 & $\mathrm{~N} / \mathrm{A}$ & $<0$ \\
\hline$N F[d B]$ & 2.8 & 10 & 19 & 3 & 5.8 & 2 \\
\hline Gain [dB] & +25 & +3.5 & 0 & +70 & +55.8 & +70 \\
\hline BW [MHz] & 8 & 20 & 60 & $0.4-30$ & 4 & $\mathrm{~N} / \mathrm{A}$ \\
\hline Ripple [dB] & $<0.8$ & $<0.4$ & 0.7 & - & - & . \\
\hline Filter order & 6 & 4 & 6 & $2 @ R F$ & 6 & $2 @ R F$ \\
\hline Stop-band rejection $[\mathrm{dB}]$ & 50 & $>55$ & $>30$ & $<15 @$ QRF & 50 & $<15 @ R F$ \\
\hline Power [mW] & $15-42 \mathrm{~mA}$ & 12.8-21.4 & 17.5 & $30-55 \mathrm{~mA}$ & $21 \mathrm{~mA}$ & $27.60 \mathrm{~mA}$ \\
\hline Area $\left[\mathrm{mm}^{2}\right]$ & 0.27 & 0.12 & 3.51 & 2 & 0.76 & 1.2 \\
\hline
\end{tabular}

\section{Fig.6: Comparison table}




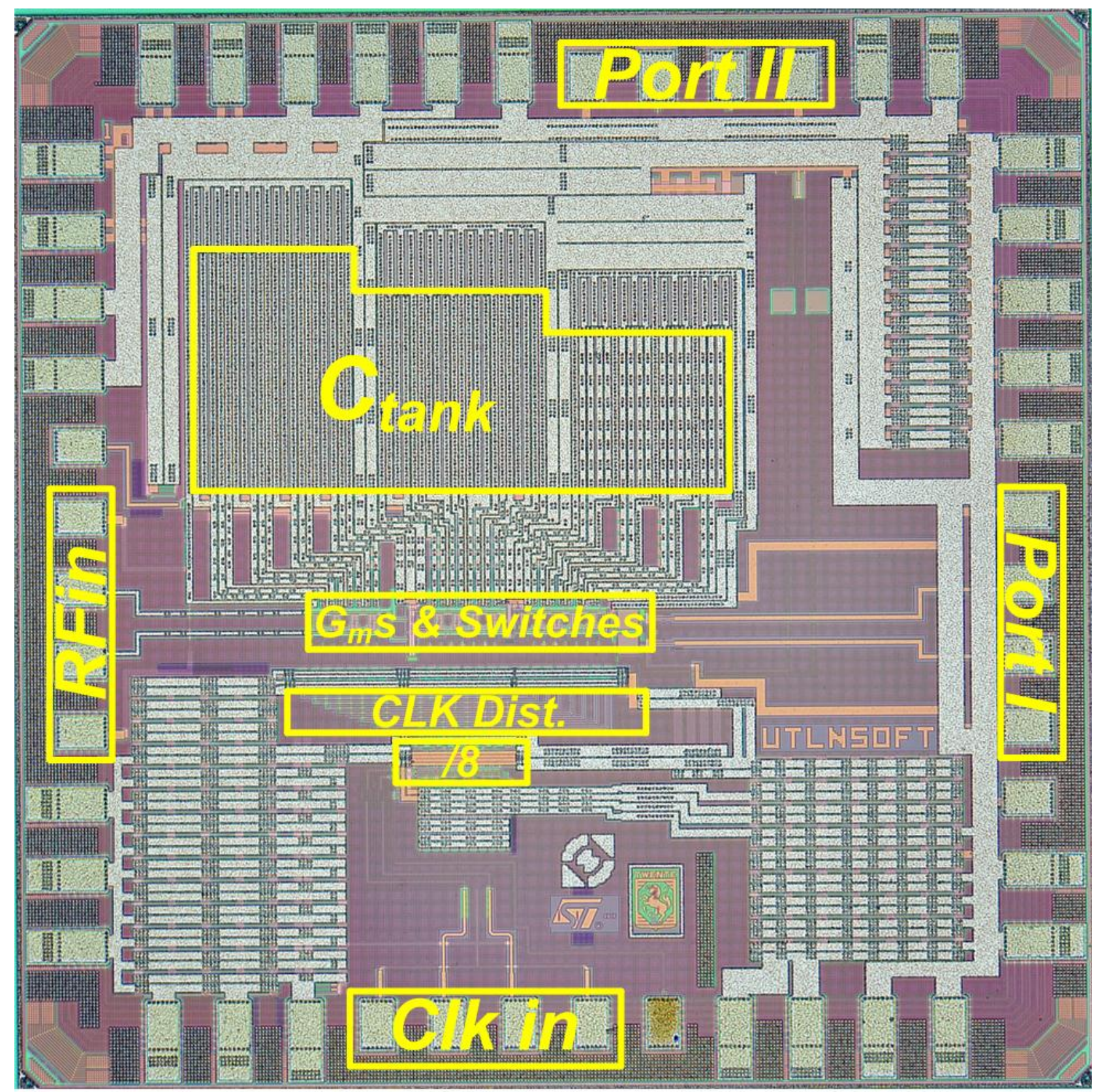

\section{Fig.7: Chip micrograph}

